\title{
Recent Results Addressing the KARMEN Timing Anomaly
}

\author{
E. D. Zimmerman \\ Columbia University, New York, New York
}

\begin{abstract}
Recent resuls from experiments at Fermilab and the Paul Scherrer Institute have constrained the parameter space available for a hypothetical particle $Q^{0}$ produced in the decay $\pi^{+} \rightarrow \mu^{+} Q^{0}$. This decay has been invoked to explain a peculiar feature of an event arrival time distribution observed in the KARMEN neutrino experiment.
\end{abstract}

\section{THE KARMEN SIGNAL}

In 1995, the KARMEN collaboration at the Rutherford Appleton Laboratory's ISIS spallation source published evidence [1] for an anomaly in the arrival-time distribution of neutrinos from pion and muon decay in a pulsed beam-stop source. The anomaly consisted of an excess of events with a small amount of electromagnetic energy $(<\sim 35 \mathrm{MeV})$ delayed $3.6 \mu$ s with respect to the beam arrival.

In KARMEN's first data set (1990-95) the anomaly comprised $83 \pm 28$ events; data through 1998 [2] increased the excess to $103 \pm 34$ events.

\section{The particle interpretation}

An explanation of the anomaly is that an exotic particle, referred to in this paper as $Q^{0}$, is produced by the rare decay $\pi^{+} \rightarrow \mu^{+} Q^{0}$ near the kinematic threshold for that process. From a $\pi^{+}$decay at rest, the $Q^{0}$ would travel 17.5 meters to the KARMEN detector in $3.6 \mu$ s (corresponding to a velocity $v \approx 0.016 c$ ). The anomalous signal in the detector would be due to the decay of the $Q^{0}$ to an electromagnetic final state. Based on the measured time of flight, the mass of the $Q^{0}$ must be $33.91 \mathrm{MeV}$. The visible energy of the anomaly favors a three-body decay to an electron (or possibly photon) pair and an invisible final-state particle, likely a neutrino. A likelihood analysis [2] has shown evidence for a correlation between position and arrival time for events in the anomaly, consistent with the slow-moving particle interpretation.

If interpreted as such a particle, the KARMEN signal corresponds to a curve in $Q^{0}$ lifetime versus $B R\left(\pi^{+} \rightarrow \mu^{+}+Q^{0}\right) \cdot B R\left(Q^{0} \rightarrow\right.$ visible $)$. This branching ratio 
is minimized at $\sim 10^{-16}$ for a lifetime $\tau_{Q^{0}}=3.6 \mu \mathrm{s}$. For any larger branching ratio, two solutions exist, one at a longer and one at a shorter lifetime. A previous experiment at the Paul Scherrer Institute (PSI) has ruled out at 90\% C.L any exotic $\pi^{+}$decays to muons above a branching ratio of $2.1 \times 10^{-8}$ [3]. This constrains the lifetime of the $Q^{0}$ to be between $\sim 10^{-7}$ and $\sim 10^{3} \mathrm{~s}$ (see Figure 1 ).

\section{Theoretical explanations}

Most theoretical speculation on the anomaly has been along one of two lines: neutral heavy leptons $[4,5]$ and light neutralinos [8]. Both are discussed briefly below:

A "standard" neutral heavy lepton ("heavy" or "sterile neutrino"), which would be produced and decayed solely through mixing with $\nu_{\mu}$, is consistent with the KARMEN data alone. However, the KARMEN result would require the particle to have a relatively large mixing with $\nu_{\mu}[6,7]$; such a large mixing is not consistent with the PSI branching ratio limit. A neutral heavy lepton explanation for the $Q^{0}$ is still allowed if its production is dominated by a small mixing with $\nu_{\mu}$ and its decay is dominated by a much larger mixing with $\nu_{\tau}$.

Another scenario consistent with the KARMEN data is an $R$-parity violating neutralino decay. This scenario is allowed in an unconstrained supersymmetric model, but chargino mass limits [9] exclude a such light neutralino in models such as SUGRA, which introduce a chargino-neutralino mass relation.

\section{FERMILAB E815 (NUTEV)}

Fermilab experiment $815(\mathrm{NuTeV})$ has performed a direct search for $Q^{0}$ decay using a beam created by the decays of high-energy pions and kaons. The experiment took data during Fermilab's 1996-97 Tevatron fixed target run, accumulating $2.54 \times$ $10^{18} 800 \mathrm{GeV}$ protons on a $\mathrm{BeO}$ target with the detector configured to search for exotic particle decays. Sign-selected secondary pions and kaons in the $100-400 \mathrm{GeV}$ range were focused down a $440 \mathrm{~m}$ decay pipe, where they could decay before hitting a steel beam dump. A total of $(1.4 \pm 0.1) \times 10^{15}$ pion decays occurred in the pipe. Neutral weakly-interacting decay products (neutrinos and possibly $Q^{0}$ 's) traveled through approximately $900 \mathrm{~m}$ of earth berm shielding before arriving at the detector.

The E815 detector consisted of an instrumented decay channel followed by an iron-target neutrino detector. The decay channel was composed of an upstream charged particle veto followed by a series of helium bags totaling $34 \mathrm{~m}$ in length, interspersed with $3 \mathrm{~m} \times 3 \mathrm{~m}$ multiwire argon-ethane drift chambers. The chambers were used to track charged particles from decays in the helium fiducial volume. The neutrino detector, which consisted of iron plates interspersed with drift chambers and liquid scintillator counters, provided calorimetry, particle identification, and 
triggering. A toroid spectrometer at the downstream end of the detector measured the momentum of muons too energetic to range out in the calorimeter.

In this experiment, the experimental signature of $Q^{0}$ decay was the spontaneous appearence of a low-mass, low-transverse momentum electron-positron pair in the helium decay channel. The analysis requirements were that two well-reconstructed tracks form a vertex in the decay channel fiducial volume, and that the tracks be identified as electrons based on the shape of their shower in the calorimeter. Because of the low mass of the $Q^{0}$, signal events typically had a very small opening angle and thus formed a single merged electron-like cluster in the calorimeter.

Backgrounds to the $Q^{0}$ search were primarily due to interactions between the high-flux neutrino beam and material in the upstream berm, veto, and decay channel drift chambers. Interactions in the berm and veto wall could produce photons or neutral kaons which could then convert or decay to charged particles in the decay channel. Interactions in the drift chambers themselves could produce charged tracks directly in the fiducial volume. These backgrounds were reduced by removing events with activity in the upstream veto wall, by making tight cuts on electron identification, and by a series of kinematic cuts designed to discriminate against the high- $Q^{2}$ events with large invariant mass final states typical of neutrino deep inelastic scattering.

With the final requirements, the expected background level from all sources was $(0.06 \pm 0.05)$ events with a signal acceptance of $16 \%$ for decays in the fiducial region. After a blind analysis, the signal region contained no events. The result excluded the short-lifetime solution to the KARMEN anomaly above a branching ratio of $\approx 5 \times 10^{-12}$ at $90 \%$ C.L. (see Figure 1 and Ref. [10]).

\section{THE SEARCH AT PSI}

A new indirect search for $Q^{0}$ production was recently conducted at PSI by M. Daum et al. [11]. The experiment exploited the near-zero $Q$-value in the $\pi^{+} \rightarrow$ $\mu^{+} Q^{0}$ decay by searching for muons from $\pi^{+}$decay emerging parallel to and with the same velocity as the parent $\pi^{+}$.

The experiment began with a proton beam aimed at a production target; a narrow-band beam of pions with momentum $150 \mathrm{MeV} / c$ was selected and focused toward a decay region. Downstream of the decay region a beamline selected charged particles of a particular momentum traveling in the forward direction. A series of scintillation counters in the downstream beamline measured the velocity of particles in the beamline, allowing identification of decay products.

The signature of $Q^{0}$ production is an excess of forward muons with a momentum of $113.5 \mathrm{MeV}$. The search technique was to scan the analysis beamline to select different momenta around $113.5 \mathrm{MeV} / c$, in $0.5 \mathrm{MeV} / c$ steps. The number of forward muons was counted in each momentum configuration, and the dependence of the muon rate was fit to a $Q^{0}$ plus background distribution.

The final fit indicated a branching ratio $B R\left(\pi^{+} \rightarrow \mu^{+} Q^{0}\right)=(1.3 \pm 2.3) \times 10^{-10}$, 


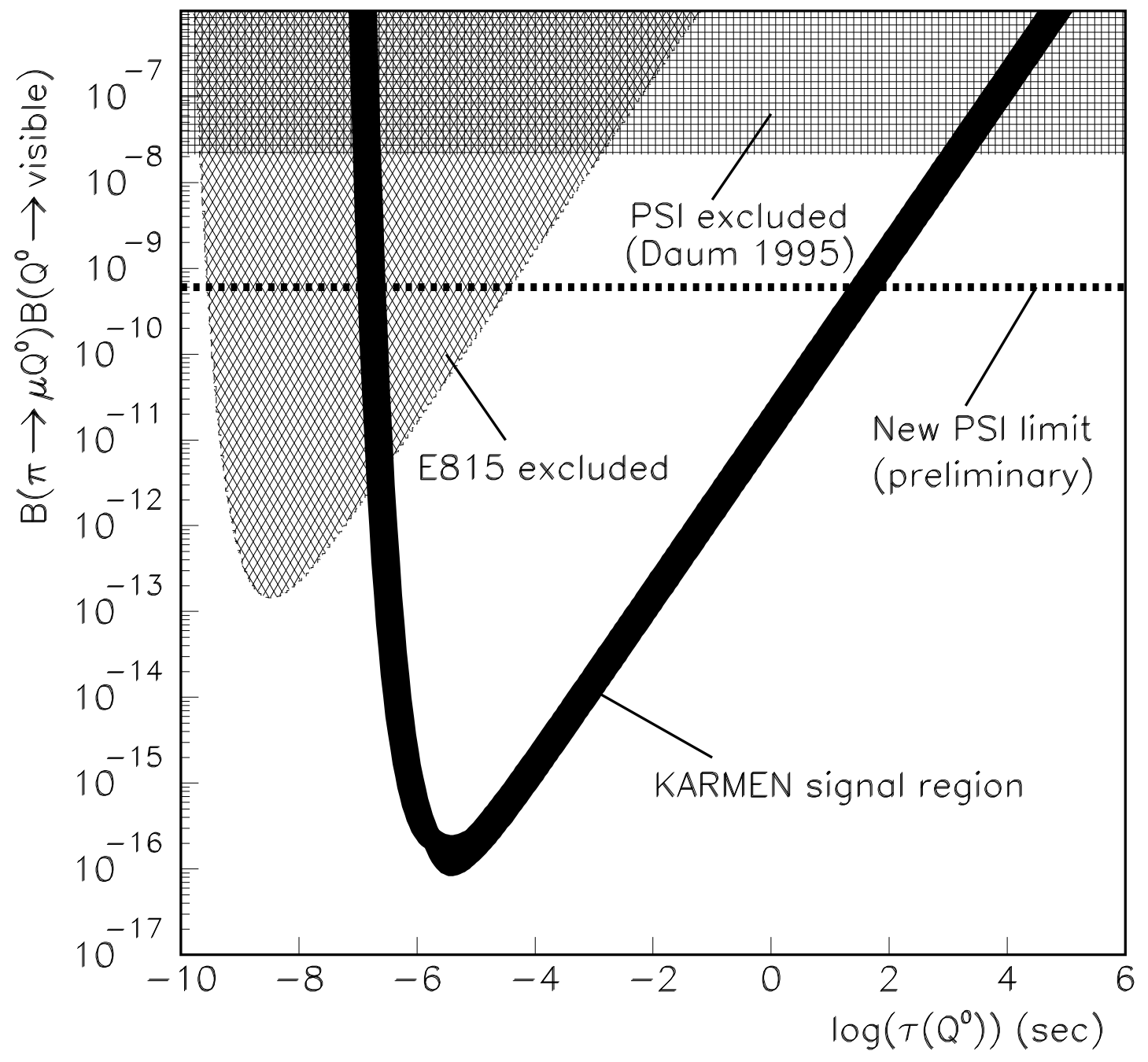

FIGURE 1. The KARMEN signal region and limits from other experiments. 
reported as a limit $B R\left(\pi^{+} \rightarrow \mu^{+} Q^{0}\right)<6.0 \times 10^{-10}$ at $95 \%$ C.L. Because the $Q^{0}$ decay is not detected, this limit applies to both the short and long lifetime solutions of the KARMEN anomaly. Unlike the E815 limit, it is independent of $B R\left(Q^{0} \rightarrow\right.$ visible $)$.

\section{STATUS OF THE ANOMALY}

At present, the timing anomaly remains an apparently significant feature of the KARMEN data set. New data from KARMEN over the next year will be interesting, but will not be a major addition to the existing data. Neither the PSI nor the Fermilab groups expect to take more data. Their current results are already approaching inherent limitations in their technique: the unavailability of arbitrarily high beam intensities in the Fermilab case, and the intrinsic $\pi^{+} \rightarrow \mu^{+} \nu \gamma$ background at PSI. Some upcoming experiments, including BooNE (Fermilab E898) will have some sensitivity to the $Q^{0}$, particularly in the short-lifetime region. A dedicated search for $Q^{0}$ decay has been proposed at ISIS, and it is possible that the muon source at a muon storage ring may be used to search for $Q^{0}$ production. No experiment currently approved is likely to have sufficient sensitivity to confirm or rule out the existence of the $Q^{0}$.

\section{ACKNOWLEDGMENTS}

The author thanks the E815 (NuTeV) collaboration, M. Daum and J. Koglin of PSI, and C. Oehler of the University of Karlsruhe. This work was supported by the National Science Foundation.

\section{REFERENCES}

1. B. Armbruster et al., Phys. Lett. B348, 19 (1995).

2. C. Oehler, Nucl. Phys. B, Proc. Suppl. 85, 101 (2000).

3. M. Daum et al., Phys. Lett. B361 179 (1995).

4. J. Govaerts, J. Deutsch, and P.M. Van Hove, Phys. Lett. B389 700 (1996).

5. V. Barger, R.J.N. Phillips, and S. Sarkar, Phys. Lett. B352 365 (1995) and erratum: Phys. Lett. B356 617 (1995).

6. M.Gronau, C.N. Leung, and J.L. Rosner, Phys. Rev. D29 2539 (1984).

7. R. E. Shrock, Phys. Rev. D24 1232 (1981).

8. D. Choudhury et al., Phys. Rev. D61 095009 (2000).

9. D. E. Groom et al., Eur. Phys. J. C15 1 (2000).

10. J. Formaggio et al., Phys. Rev. Lett. 844043 (2000).

11. J. Koglin, PhD Dissertation, The University of Virginia (2000). 